\title{
Space of Functions with Some Generalization of Bounded Variation in the Sense of de La Vallée Poussin
}

\author{
René Erlín Castillo, ${ }^{1}$ Humberto Rafeiro, ${ }^{2}$ and Eduard Trousselot ${ }^{3}$ \\ ${ }^{1}$ Departamento de Matemáticas, Universidad Nacional de Colombia, Bogotá, Colombia \\ ${ }^{2}$ Departamento de Matemáticas, Pontificia Universidad Javeriana, Carrera 7a No. 43-82, Bogotá, Colombia \\ ${ }^{3}$ Departamento de Matemáticas, Universidad de Oriente, Cumaná 6101, Estado Sucre, Venezuela \\ Correspondence should be addressed to René Erlín Castillo; recastillo@unal.edu.co
}

Received 22 January 2015; Accepted 1 April 2015

Academic Editor: Henryk Hudzik

Copyright (C) 2015 René Erlín Castillo et al. This is an open access article distributed under the Creative Commons Attribution License, which permits unrestricted use, distribution, and reproduction in any medium, provided the original work is properly cited.

We introduce a function space with some generalization of bounded variation in the sense of de la Vallée Poussin and study some of its properties, like embeddings and decompositions, among others.

\section{Introduction}

Two centuries ago, around 1880, Jordan (see [1]) introduced the notion of a function of bounded variation and established the relation between these functions and monotonic ones when he was studying convergence of Fourier series. Later on the concept of bounded variation was generalized in various directions by many mathematicians, such as L. Ambrosio, R. Caccioppoli, L. Cesari, E. Conway, G. Dal Maso, E. de Giorgi, S. Hudjaev, J. Musielak, O. Oleinik, W. Orlicz, F. Riesz, J. Smoller, L. Tonelli, A. Vol'pert, and N. Wiener, among many others. It is noteworthy to mention that many of these generalizations were motivated by problems in such areas as calculus of variations, convergence of Fourier series, geometric measure theory, and mathematical physics. For many applications of functions of bounded variation in mathematical physics see the monograph [2]. For a thorough exposition regarding bounded variation spaces and related topics, see the recent monograph [3].

For recent generalization of the concept of bounded variation, see [4-6].

In 1908 de La Vallée Poussin [7] generalized the concept of bounded variation functions. He defined the second bounded variation of a function $f$ in the interval $[a, b]$ by

$$
\begin{aligned}
& \mathrm{V}^{2}(f) \\
& \quad=\mathrm{V}^{2}(f,[a, b])
\end{aligned}
$$

$$
=\sup _{\Pi} \sum_{j=1}^{n-1}\left|\frac{f\left(t_{j+1}\right)-f\left(t_{j}\right)}{t_{j+1}-t_{j}}-\frac{f\left(t_{j}\right)-f\left(t_{j-1}\right)}{t_{j}-t_{j-1}}\right|,
$$

where the supremum is taken over all partitions $\Pi=\{a=$ $\left.x_{0}<\cdots<x_{n}=b\right\}$ of the interval $[a, b]$. The function $f$ is said to be a bounded second variation function in $[a, b]$ if $\mathrm{V}^{2}(f,[a, b])<\infty$. The linear space of all such functions is denoted by $\mathrm{BV}^{2}[a, b]$. The following characterization of $\mathrm{BV}^{2}[a, b]$ was given by de La Vallée Poussin in [7].

Proposition 1. A function $f$ is of bounded second variation if and only if $f$ can be written as the difference of two convex functions.

Since any convex function $f:[a, b] \rightarrow \mathbb{R}$ has right and left derivatives on each interior point of the interval $[a, b]$ and moreover $f$ is differentiable a.e. (see [8]), then from Proposition 1 we deduce that all bounded second variation functions in $[a, b]$ have all the properties described above. The space $\mathrm{BV}^{2}[a, b]$ equipped with the norm

$$
\|f\|_{\mathrm{BV}^{2}[a, b]}=|f(a)|+\left|f^{\prime}(a)\right|+\mathrm{V}^{2}(f,[a, b])
$$

is a Banach space. These functions were generalized to bounded second variation functions with respect to a strictly 
increasing and continuous function $\alpha$ (see [9]). We introduce this concept considering usual partition (see [9]) and block partitions (see [3]). Then we show that the two definitions are related and study some of its properties.

From now on, we will always use $\alpha$ as a strictly increasing continuous function, whenever not stated otherwise.

\section{Preliminaries}

We want to recall the so-called Popoviciu variation (introduced in 1933 by Popoviciu in [10]) for a partition $\Pi=\{a=$ $\left.x_{1}<x_{2}<\cdots<x_{m}=b\right\}$ and a function $f:[a, b] \rightarrow \mathbb{R}$ given by

$$
\begin{aligned}
\operatorname{Var}_{k, 1} & (f, \Pi,[a, b]) \\
\quad & =\sum_{j=1}^{m-k+1}\left|f\left[x_{j}, \ldots, x_{j+k-1}\right]-f\left[x_{j-1}, \ldots, x_{j+k-2}\right]\right|,
\end{aligned}
$$

where $f[\cdot, \ldots, \cdot]$ is defined recursively in the following way:

$$
\begin{aligned}
& f\left[x_{0}\right]:=f\left(x_{0}\right), \\
& f\left[x_{0}, x_{1}\right]:=\frac{f\left[x_{1}\right]-f\left[x_{0}\right]}{x_{1}-x_{0}} \\
& f\left[x_{0}, x_{1}, x_{2}\right]:=\frac{f\left[x_{1}, x_{2}\right]-f\left[x_{0}, x_{1}\right]}{x_{2}-x_{0}} \\
& \vdots \\
& f\left[x_{0}, x_{1}, \ldots, x_{k}\right] \\
& :=\frac{f\left[x_{1}, x_{2}, \ldots, x_{k}\right]-f\left[x_{0}, x_{1}, \ldots, x_{k-1}\right]}{x_{k}-x_{0}} .
\end{aligned}
$$

In the following we will consider a block partition $\Pi$ of the interval $[a, b]$ which we will call partition of type II. It will be taken in the following way:

$$
\begin{aligned}
\Pi & =\left\{a=x_{1,1}<x_{1,2} \leqslant x_{1,3}<x_{1,4}=x_{2,1}<x_{2,2} \leqslant x_{2,3}\right. \\
& <x_{2,4}=x_{3,1}<\cdots<x_{n-1,4}=x_{n, 1}<x_{n, 2} \leqslant x_{n, 3}<x_{n, 4} \\
& =b\}
\end{aligned}
$$

in place of the regular partition $\Pi$ which has been used in the study of functions with some variation. In what follows we call a regular partition $\Pi=\left\{a=x_{0}<x_{1}<\cdots<x_{n}=b\right\}$ a partition of type $I$.

Definition 2. Let $f$ be a real-valued function defined on $[a, b]$ and let $\Pi$ be a type I partition of $[a, b]$. Let

$$
\sigma_{(2, \alpha)}(f, \Pi)=\sum_{j=1}^{n-2}\left|f_{\alpha}\left[x_{j}, x_{j+1}\right]-f_{\alpha}\left[x_{j+1}, x_{j+2}\right]\right|,
$$

where

$$
\begin{aligned}
f_{\alpha}[a, b] & =\frac{f(b)-f(a)}{\alpha(b)-\alpha(a)}, \\
\mathrm{V}_{(2, \alpha)}(f,[a, b]) & =\mathrm{V}_{(2, \alpha)}=\sup _{\Pi} \sigma_{(2, \alpha)}(f, \Pi),
\end{aligned}
$$

where the supremum is taken over all partitions of type I of $[a, b]$. The space $\mathrm{V}_{(2, \alpha)}(f)$ is called $(2, \alpha)$-variation in the sense of de La Vallée Poussin of type I. If $\mathrm{V}_{(2, \alpha)}(f)<\infty$ the function $f$ is said to be of $(2, \alpha)$-variation in the sense of de La Vallée Poussin of type I. The set of all such functions is denoted by $\mathrm{BV}_{(2, \alpha)}[a, b]$.

Definition 3. Let $f$ be a real-valued function defined on $[a, b]$ and let $\Pi$ be a type II partition of $[a, b]$. Let

$$
\begin{aligned}
\sigma^{(2, \alpha)}(f, \Pi) & =\sum_{j=1}^{n}\left|f_{\alpha}\left[x_{j, 1}, x_{j, 2}\right]-f_{\alpha}\left[x_{j, 3}, x_{j, 4}\right]\right|, \\
\mathrm{V}^{(2, \alpha)}(f,[a, b]) & =\mathrm{V}^{(2, \alpha)}(f)=\sup _{\Pi} \sigma^{(2, \alpha)}(f, \Pi),
\end{aligned}
$$

where the supremum is taken on all type II partition of $[a, b]$. The number $\mathrm{V}^{(2, \alpha)}(f,[a, b])$ is called the $(2, \alpha)$-variation in the sense of de La Vallée Poussin of type II in $[a, b]$. If $\mathrm{V}^{(2, \alpha)}(f,[a, b])<\infty, f$ is said to be a function of $(2, \alpha)$ variation in the sense of de La Vallée Poussin of type II. The set of all such functions is denoted by $\mathrm{BV}^{(2, \alpha)}[a, b]$.

Definition 4. A function $f:[a, b] \rightarrow \mathbb{R}$ is said to be an $\alpha$ Lipschitz function if there exists a constant $M>0$ such that

$$
|f(x)-f(y)| \leq M|\alpha(x)-\alpha(y)|
$$

for all $x, y \in[a, b]$. We define the space $\alpha$-Lip $[a, b]$ as the space of all $\alpha$-Lipschitz functions. This space is normable, via the norm

$$
\|f\|_{\alpha-\text { Lip }}:=|f(a)|+\sup _{x \neq y} \frac{|f(x)-f(y)|}{|\alpha(x)-\alpha(y)|} .
$$

We now introduce some concepts that will be of fundamental use (cf. [11]).

Definition 5. One said that a function $f$ is $\alpha$-convex in $[a, b]$ if, for $a \leqslant x \leqslant z \leqslant y \leqslant b$, one has

$$
f(z) \leqslant \frac{\alpha(z)-\alpha(x)}{\alpha(y)-\alpha(x)} f(y)+\frac{\alpha(y)-\alpha(z)}{\alpha(y)-\alpha(x)} f(x) .
$$

Definition 6. A function $f:[a, b] \rightarrow \mathbb{R}$ is said to be absolutely continuous with respect to $\alpha$ if, for every $\varepsilon>0$, there exists some $\delta>0$ such that if $\left\{\left(a_{j}, b_{j}\right)\right\}_{j=1}^{n}$ are disjoint open subintervals of $[a, b]$, then

$$
\sum_{j=1}^{n}\left|\alpha\left(b_{j}\right)-\alpha\left(a_{j}\right)\right|<\delta
$$


All functions in $\alpha-\mathrm{AC}[a, b]$ are bounded and form an algebra of functions under pointwise defined standard operations.

Definition 7. Suppose $f$ and $\alpha$ are real-valued functions defined on the same open interval $I$ and let $x_{0} \in I$. One says that $f$ is $\alpha$-differentiable at $x_{0}$ if the following limit exists:

$$
\lim _{x \rightarrow x_{0}} \frac{f(x)-f\left(x_{0}\right)}{\alpha(x)-\alpha\left(x_{0}\right)} .
$$

If the limit exists we denote its value by $f_{\alpha}^{\prime}\left(x_{0}\right)$, which we call the $\alpha$-derivative of $f$ at $x_{0}$.

By $\mu_{\alpha}$ we denote the Lebesgue-Stieltjes measure induced by $\alpha$. For a standard treatment regarding Lebesgue-Stieltjes measure and integral, see, for example, [12].

The following two results are proved in $[11,13,14]$. Theorem 8 connects the $\alpha$ - $\mathrm{AC}[a, b]$ concept with the concept of $\alpha$-derivative.

Theorem 8. Let $f \in \alpha-\mathrm{AC}[a, b]$; then $f_{\alpha}^{\prime}$ exists and is finite $\mu_{\alpha}$ a.e. on $[a, b]$. Moreover $f_{\alpha}^{\prime}$ is integrable in the Lebesgue-Stieltjes sense and

$$
f(x)=f(a)+(L S) \int_{a}^{x} f_{\alpha}^{\prime}(t) d \alpha(t), \quad x \in[a, b] .
$$

Theorem 9. Let $p$ be an increasing function and $\alpha$ a continuous function in $[a, b]$. Then one has that

$$
x \longmapsto S(x)=(L S) \int_{a}^{x} p(t) d \alpha(t)
$$

is an $\alpha$-convex function on $[a, b]$.

Lemma 10. Let $v$ be an $\alpha$-convex function and $a \leqslant \lambda \leqslant \xi \leqslant$ $\mu \leqslant b$; then

$$
\frac{v(\xi)-v(\lambda)}{\alpha(\xi)-\alpha(\lambda)} \leqslant \frac{v(\mu)-v(\lambda)}{\alpha(\mu)-\alpha(\lambda)} \leqslant \frac{v(\mu)-v(\xi)}{\alpha(\mu)-\alpha(\xi)}
$$

that is, $v_{\alpha}[\lambda, \xi] \leqslant v_{\alpha}[\lambda, \mu] \leqslant v_{\alpha}[\xi, \mu]$ with $v_{\alpha}[p, q]=(v(q)-$ $v(p)) /(\alpha(q)-\alpha(p))$.

\section{Equivalent Definitions}

In this section we show that $\mathrm{BV}_{(2, \alpha)}[a, b]=\mathrm{BV}^{(2, \alpha)}[a, b]$. In order to do that, we gather several previous results.

Lemma 11. Let $f$ be defined on $[a, b]$ and let $\Pi_{1}=\left\{a=x_{1}<\right.$ $\left.x_{2}<x_{3}=b\right\}$ be a partition of $[a, b]$. Then there exists $\lambda$ and $\mu$ in $[0,1]$ with $\lambda+\mu=1$ such that

$$
f_{\alpha}\left[x_{1}, x_{3}\right]=\lambda f_{\alpha}\left[x_{1}, x_{2}\right]+\mu f_{\alpha}\left[x_{2}, x_{3}\right]
$$

Proof. Observe that

$$
\begin{aligned}
f_{\alpha}\left[x_{1}, x_{3}\right]= & \frac{\alpha\left(x_{3}\right)-\alpha\left(x_{2}\right)}{\alpha\left(x_{3}\right)-\alpha\left(x_{1}\right)}\left[\frac{f\left(x_{3}\right)-f\left(x_{2}\right)}{\alpha\left(x_{3}\right)-\alpha\left(x_{2}\right)}\right] \\
& +\frac{\alpha\left(x_{2}\right)-\alpha\left(x_{1}\right)}{\alpha\left(x_{3}\right)-\alpha\left(x_{1}\right)}\left[\frac{f\left(x_{2}\right)-f\left(x_{1}\right)}{\alpha\left(x_{2}\right)-\alpha\left(x_{1}\right)}\right] \\
= & \lambda f_{\alpha}\left[x_{1}, x_{3}\right]+\mu f_{\alpha}\left[x_{2}, x_{3}\right],
\end{aligned}
$$

where $\lambda$ and $\mu$ are straightforward from the equation.

The following result shows that the number $\sigma_{(2, \alpha)}(f, \Pi)$ does not decrease if we add points in partition $\Pi$.

Theorem 12. Let $f$ be defined on $[a, b]$ and let $\Pi=\left\{a=x_{1}<\right.$ $\left.\cdots<x_{s-1}<x_{s}<\cdots<x_{n-1}<x_{n}=b\right\}$ be a partition of type $I$ and $y \in\left(x_{s-1}, x_{s}\right)$ with $s=2, \ldots, n$. Let one consider

$$
\begin{aligned}
\Pi^{\prime} & =\left\{a=x_{1}<\cdots<x_{s-2}<x_{s-1}<y<x_{s}<x_{s+1}<\cdots\right. \\
& \left.<x_{n-1}<x_{n}=b\right\} .
\end{aligned}
$$

Then

$$
\sigma_{(2, \alpha)}(f, \Pi) \leqslant \sigma_{(2, \alpha)}\left(f, \Pi^{\prime}\right)
$$

Proof.

Case $1(2<s<n)$. Let us consider all the terms of $\sigma_{(2, \alpha)}(f, \Pi)$ on which $y$ play a role; we have to estimate the expression

$$
\begin{aligned}
& \left|f_{\alpha}\left[x_{s-2}, x_{s-1}\right]-f_{\alpha}\left[x_{s-1}, x_{s}\right]\right| \\
& \quad+\left|f_{\alpha}\left[x_{s-1}, x_{s}\right]-f_{\alpha}\left[x_{s}, x_{s+1}\right]\right| .
\end{aligned}
$$

Applying Lemma 11 in order to introduce the point $y$ in $\left(x_{s-1}, x_{s}\right)$, we obtain that there exists positive $\lambda$ and $\mu$ with $\lambda+\mu=1$ such that

$$
f_{\alpha}\left[x_{s-1}, x_{s}\right]=\lambda f_{\alpha}\left[x_{s-1}, y\right]+\mu f_{\alpha}\left[y, x_{s}\right]
$$

replacing in (21) and using the fact that $\lambda+\mu=1$ we have

$$
\begin{aligned}
& \left|f_{\alpha}\left[x_{s-2}, x_{s-1}\right]-(1-\mu) f_{\alpha}\left[x_{s-1}, y\right]-\mu f_{\alpha}\left[y, x_{s}\right]\right| \\
& \quad+\mid \lambda f_{\alpha}\left[x_{s-1}, y\right]+(1-\lambda) f_{\alpha}\left[y, x_{s}\right] \\
& \quad-f_{\alpha}\left[x_{s}, x_{s+1}\right] \mid .
\end{aligned}
$$

From the triangle inequality in (23) we obtain

$$
\begin{aligned}
\leqslant & \left|f_{\alpha}\left[x_{s-2}, x_{s-1}\right]-f_{\alpha}\left[x_{s-1}, y\right]\right| \\
& +\left|f_{\alpha}\left[x_{s-1}, y\right]-f_{\alpha}\left[y, x_{s}\right]\right| \\
& +\left|f_{\alpha}\left[y, x_{s}\right]-f_{\alpha}\left[x_{s}, x_{s+1}\right]\right| .
\end{aligned}
$$

That is, the expression in (21) is less than or equal to (24). Since the remaining terms in $\sigma_{(2, \alpha)}(f, \Pi)$ and $\sigma_{(2, \alpha)}\left(f, \Pi^{\prime}\right)$ coincide, we get (20). 
Case $2(s=2)$. Let $y \in\left(x_{1}, x_{2}\right)$; in this case we have a unique term of $\sigma_{(2, \alpha)}(f, \Pi)$, where the interval $\left(x_{1}, x_{2}\right)$ plays a role; applying Lemma 11 we obtain $f_{\alpha}\left[x_{1}, x_{2}\right]=\lambda f_{\alpha}\left[x_{1}, y\right]+(1-$ d) $f_{\alpha}\left[y, x_{2}\right]$ with $0<\lambda<1$. Hence

$$
\begin{aligned}
\mid f_{\alpha} & {\left[x_{1}, x_{2}\right]-f_{\alpha}\left[x_{2}, x_{3}\right] \mid } \\
= & \left|\lambda f_{\alpha}\left[x_{1}, y\right]+(1-\lambda) f_{\alpha}\left[y, x_{2}\right]-f_{\alpha}\left[x_{2}, x_{3}\right]\right| \\
\leqslant & \lambda\left|f_{\alpha}\left[x_{1}, y\right]-f_{\alpha}\left[y, x_{2}\right]\right| \\
& +\left|f_{\alpha}\left[y, x_{2}\right]-f_{\alpha}\left[x_{2}, x_{3}\right]\right| \\
\leqslant & \left|f_{\alpha}\left[x_{1}, y\right]-f_{\alpha}\left[y, x_{2}\right]\right| \\
& +\left|f_{\alpha}\left[y, x_{2}\right]-f_{\alpha}\left[x_{2}, x_{3}\right]\right|
\end{aligned}
$$

and since the remaining terms of $\sigma_{(2, \alpha)}(f, \Pi)$ and $\sigma_{(2, \alpha)}\left(f, \Pi^{\prime}\right)$ coincide, we obtain (20).

Case $3(s=n)$. This case is similar to Case 2 .

Corollary 13. Let $f$ be a function defined in $[a, b]$ and let $\Pi^{\prime}$ be a refinement of partition $\Pi$. Then

$$
\sigma_{(2, \alpha)}(f, \Pi) \leqslant \sigma_{(2, \alpha)}\left(f, \Pi^{\prime}\right) .
$$

Proof. The proof follows from Theorem 12 by induction.

Theorem 14. Let $f$ be defined on $[a, b]$. Then

$$
\mathrm{V}^{(2, \alpha)}(f) \leqslant \mathrm{V}_{(2, \alpha)}(f) \leqslant 7 \mathrm{~V}^{(2, \alpha)}(f) .
$$

Therefore

$$
\mathrm{BV}_{(2, \alpha)}[a, b]=\mathrm{BV}^{(2, \alpha)}[a, b]
$$

Proof. Consider the following.

Case 1. Let $f \in \mathrm{BV}_{(2, \alpha)}[a, b]$ and $\Pi$ be a type II partition of $[a, b]$. First we assume that $x_{j, 2}<x_{j, 3}$ for all $j$. On each $\sigma^{(2, \alpha)}(f, \Pi)$ we may apply the triangle inequality for $j=$ $1, \ldots, n$ to obtain

$$
\begin{aligned}
& \left|f_{\alpha}\left[x_{j, 1}, x_{j, 2}\right]-f_{\alpha}\left[x_{j, 3}, x_{j, 4}\right]\right| \\
& \leqslant\left|f_{\alpha}\left[x_{j, 1}, x_{j, 2}\right]-f_{\alpha}\left[x_{j, 2}, x_{j, 3}\right]\right| \\
& \quad+\left|f_{\alpha}\left[x_{j, 2}, x_{j, 3}\right]-f_{\alpha}\left[x_{j, 3}, x_{j, 4}\right]\right| .
\end{aligned}
$$

Then

$$
\begin{aligned}
\sigma^{(2, \alpha)}(f, \Pi) \leqslant & \sum_{j=1}^{n}\left|f_{\alpha}\left[x_{j, 1}, x_{j, 2}\right]-f_{\alpha}\left[x_{j, 2}, x_{j, 3}\right]\right| \\
& +\sum_{j=1}^{n}\left|f_{\alpha}\left[x_{j, 2}, x_{j, 3}\right]-f_{\alpha}\left[x_{j, 3}, x_{j, 4}\right]\right| .
\end{aligned}
$$

Adding all of those terms we have

$$
\begin{aligned}
\sigma^{(2, \alpha)}(f, \Pi) \leqslant & \sum_{j=1}^{n-1}\left|f_{\alpha}\left[x_{j, 1}, x_{j, 2}\right]-f_{\alpha}\left[x_{j, 2}, x_{j, 3}\right]\right| \\
& +\left|f_{\alpha}\left[x_{n, 1}, x_{n, 2}\right]-f_{\alpha}\left[x_{n, 2}, x_{n, 3}\right]\right| \\
& +\sum_{j=1}^{n-1}\left|f_{\alpha}\left[x_{j, 2}, x_{j, 3}\right]-f_{\alpha}\left[x_{j, 3}, x_{j, 4}\right]\right| \\
& +\left|f_{\alpha}\left[x_{n, 2}, x_{n, 3}\right]-f_{\alpha}\left[x_{n, 3}, x_{n, 4}\right]\right| .
\end{aligned}
$$

This last sum is bounded by $\sigma_{(2, \alpha)}(f, \Pi)$ if we consider $\Pi$ as a type I partition; thus

$$
\sigma^{(2, \alpha)}(f, \Pi) \leqslant \sigma_{(2, \alpha)}(f, \Pi) \leqslant \mathrm{V}_{(2, \alpha)}(f)<\infty .
$$

In the case $x_{j, 2}=x_{j, 3}$ for some $j$, we do not need to apply the triangle inequality, since $\left|f_{\alpha}\left[x_{j, 1}, x_{j, 2}\right]-f_{\alpha}\left[x_{j, 3}, x_{j, 4}\right]\right|=$ $\left|f_{\alpha}\left[x_{j, 1}, x_{j, 2}\right]-f_{\alpha}\left[x_{j, 2}, x_{j, 4}\right]\right| . \sigma^{(2, \alpha)}(f, \Pi)$ is bounded by

$$
\sigma^{(2, \alpha)}(f, \Pi) \leqslant \Sigma^{\prime}+\Sigma,
$$

where $\Sigma^{\prime}$ is the summation with the terms $x_{j, 2}=x_{j, 3}$ and $\Sigma$ is the summation where $x_{j, 2}<x_{j, 3}$.

Since (32) holds for all type II partitions, we conclude that $\mathrm{V}^{(2, \alpha)}(f) \leqslant \mathrm{V}_{(2, \alpha)}(f)<\infty$, implying that $f \in \mathrm{BV}^{(2, \alpha)}[a, b]$.

Case 2. Conversely, let $f \in \mathrm{BV}^{(2, \alpha)}[a, b]$ and $\Pi$ be a type I partition of $[a, b]$; that is, $\Pi=\left\{a=x_{1}<x_{2}<\cdots<x_{n}=\right.$ $b\}$. Eventually adding one or two points we can suppose that $n=3 p$, since the addition of points in the partition does not decrease the sum $\sigma_{(2, \alpha)}$ (Theorem 12). Hence

$$
\begin{aligned}
\sigma_{(2, \alpha)} & (f, \Pi) \\
= & \sum_{j=1}^{3 p-2}\left|f_{\alpha}\left[x_{j}, x_{j+1}\right]-f_{\alpha}\left[x_{j+1}, x_{j+2}\right]\right| \\
= & \sum_{k=0}^{p-2} \sum_{j=3 k+1}^{3 k+3}\left|f_{\alpha}\left[x_{j}, x_{j+1}\right]-f_{\alpha}\left[x_{j+1}, x_{j+2}\right]\right| \\
& +\left|f_{\alpha}\left[x_{3 p-2}, x_{3 p-1}\right]-f_{\alpha}\left[x_{3 p-1}, x_{3 p}\right]\right|,
\end{aligned}
$$

where we group the terms in threes.

On each interval $\left(x_{j}, x_{j+1}\right)$ we introduce two points: $x_{j}<$ $x_{j, 1}<x_{j, 2}<x_{j+1}$ for $j=3, \ldots, n-1$. On each term from the sum we apply the triangle inequality (for the last term we give a different treatment) to obtain

$$
\begin{aligned}
& \left|f_{\alpha}\left[x_{j}, x_{j+1}\right]-f_{\alpha}\left[x_{j+1}, x_{j+2}\right]\right| \\
& \leqslant\left|f_{\alpha}\left[x_{j}, x_{j+1}\right]-f_{\alpha}\left[x_{j+2,1}, x_{j+2,2}\right]\right| \\
& \quad+\left|f_{\alpha}\left[x_{j+1}, x_{j+2}\right]-f_{\alpha}\left[x_{j+2,1}, x_{j+2,2}\right]\right|
\end{aligned}
$$


for $j=1, \ldots, n-3$. We introduce these terms in $\sigma_{(2, \alpha)}(f, \Pi)$ to get

$$
\begin{aligned}
& \sigma_{(2, \alpha)}(f, \Pi) \\
& =\sum_{k=1}^{3}\left(\sum_{j=0}^{p-2}\left|f_{\alpha}\left[x_{3 j+k}, x_{3 j+k+1}\right]-f_{\alpha}\left[x_{3 j+k+2,1}, x_{3 j+k+2,2}\right]\right|\right) \\
& +\sum_{k=1}^{3}\left(\sum_{j=0}^{p-2}\left|f_{\alpha}\left[x_{3 j+k+1}, x_{3 j+k+2}\right]-f_{\alpha}\left[x_{3 j+k+2,1}, x_{3 j+k+2,2}\right]\right|\right) \\
& +\left|f_{\alpha}\left[x_{n-2}, x_{n-1}\right]-f_{\alpha}\left[x_{n-1}, x_{n}\right]\right| .
\end{aligned}
$$

A partition of type II is obtained in each summation in parenthesis and therefore it is bounded by $\mathrm{V}^{(2, \alpha)}(f)$; accordingly

$$
\begin{aligned}
\sigma_{(2, \alpha)}(f, \Pi) \leqslant & 6 \mathrm{~V}^{(2, \alpha)}(f) \\
& +\left|f_{\alpha}\left[x_{n-2}, x_{n-1}\right]-f_{\alpha}\left[x_{n-1}, x_{n}\right]\right| .
\end{aligned}
$$

The term $\left|f_{\alpha}\left[x_{n-2}, x_{n-1}\right]-f_{\alpha}\left[x_{n-1}, x_{n}\right]\right|$ is bounded by $\mathrm{V}^{(2, \alpha)}(f)$. Finally $\sigma_{(2, \alpha)}(f) \leqslant 7 \mathrm{~V}^{(2, \alpha)}(f)<\infty$. Since this holds for all partitions of type I we conclude that $\mathrm{V}_{(2, \alpha)}(f) \leqslant$ $7 \mathrm{~V}^{(2, \alpha)}(f)<\infty$ and this $f \in \mathrm{BV}_{(2, \alpha)}[a, b]$.

Remark 15. This result guarantees that the $\mathrm{BV}^{(2, \alpha)}[a, b]$ functions satisfy all the properties verified by the $\mathrm{BV}_{(2, \alpha)}[a, b]$ functions and reciprocally. In the coming demonstrations we choose the partition that will be more appropriate.

\section{4. $\mathrm{BV}^{(2, \alpha)}=\mathrm{BV}_{(2, \alpha)}$ as Linear Spaces}

First of all we will prove some embedding between sets, to conclude that $\mathrm{BV}^{(2, \alpha)}[a, b] \subset \mathrm{B}[a, b]$, where $\mathrm{B}[a, b]$ stands for the set of bounded functions, and therefore we may consider linear subspaces.

In the following lemma we will demonstrate that all terms of the form $f_{\alpha}[p, q]$ are bounded.

Lemma 16. If $f \in \mathrm{BV}_{(2, \alpha)}[a, b]=\mathrm{BV}^{(2, \alpha)}[a, b]$, then there exists $M>0$ such that, for all $x_{1}, x_{2}$ with $a \leqslant x_{1}<x_{2} \leqslant b$, it holds that

$$
\left|\frac{f\left(x_{2}\right)-f\left(x_{1}\right)}{\alpha\left(x_{2}\right)-\alpha\left(x_{1}\right)}\right|=\left|f_{\alpha}\left[x_{1}, x_{2}\right]\right| \leqslant M \text {. }
$$

Proof. Fix $a_{1}$ and $a_{2}$ such that $a<a_{1}<a_{2}<b$ and

$$
\left|\frac{f\left(a_{2}\right)-f\left(a_{1}\right)}{\alpha\left(a_{2}\right)-\alpha\left(a_{1}\right)}\right|=\left|f_{\alpha}\left[a_{1}, a_{2}\right]\right|=A .
$$

Case $1\left(x_{2}<b\right)$. Let $x_{3}$ and $x_{4}$ be points such that $\max \left\{x_{2}, a_{2}\right\}<x_{3}<x_{4} \leqslant b$; then $a_{1}<a_{2}<x_{3}<x_{4}$ and $x_{1}<x_{2}<x_{3}<x_{4}$ are points in partitions of type II of $[a, b]$. Therefore $\left|f_{\alpha}\left[a_{1}, a_{2}\right]-f_{\alpha}\left[x_{3}, x_{4}\right]\right| \leqslant \mathrm{V}^{(2, \alpha)}(f)$ and $\left|f_{\alpha}\left[x_{1}, x_{2}\right]-f_{\alpha}\left[x_{3}, x_{4}\right]\right| \leqslant \mathrm{V}^{(2, \alpha)}(f)$. Using the triangle inequality we have

$$
\begin{aligned}
\left|f_{\alpha}\left[x_{1}, x_{2}\right]\right| \leqslant & \left|f_{\alpha}\left[x_{1}, x_{2}\right]-f_{\alpha}\left[x_{3}, x_{4}\right]\right| \\
& +\left|f_{\alpha}\left[x_{3}, x_{4}\right]-f_{\alpha}\left[a_{1}, a_{2}\right]\right| \\
& +\left|f_{\alpha}\left[a_{1}, a_{2}\right]\right| \leqslant 2 \mathrm{~V}^{(2, \alpha)}(f)+A \\
= & M_{1} .
\end{aligned}
$$

Case $2\left(x_{2}=b\right.$ and $\left.a<x_{1}\right)$. Let $x_{3}, x_{4}$ be points such that $a \leqslant x_{3}<x_{4}<\min \left\{a_{1}, x_{1}\right\}$; then $x_{3}<x_{4}<a_{1}<$ $a_{2}$ and $x_{3}<x_{4}<x_{1}<x_{2}$ are partitions of type II of $[a, b]$. Therefore $\left|f_{\alpha}\left[x_{3}, x_{4}\right]-f_{\alpha}\left[a_{1}, a_{2}\right]\right| \leqslant \mathrm{V}^{(2, \alpha)}(f)$ and $\left|f_{\alpha}\left[x_{3}, x_{4}\right]-f_{\alpha}\left[x_{1}, x_{2}\right]\right| \leqslant \mathrm{V}^{(2, \alpha)}(f)$. Similarly as in Case 1 we obtain $\left|f_{\alpha}\left[x_{1}, x_{2}\right]\right| \leqslant M_{1}$.

Case $3\left(x_{1}=a\right.$ and $\left.x_{2}=b\right)$. Consider

$$
\left|f_{\alpha}\left[x_{1}, x_{2}\right]\right|=\left|f_{\alpha}[a, b]\right|=\left|\frac{f(b)-f(a)}{\alpha(b)-\alpha(a)}\right|=M_{2} \text {. }
$$

Taking $M=\max \left\{M_{1}, M_{2}\right\}$ we deduce that for all $x_{1}, x_{2}$ such that $a \leqslant x_{1}<x_{2} \leqslant b$ we have $\left|f_{\alpha}\left[x_{1}, x_{2}\right]\right| \leqslant M$.

This allow us to prove that all $(2, \alpha)$-variation function in $[a, b]$ is $\alpha$-Lipschitz in $[a, b]$.

Theorem 17. If $f$ is a $(2, \alpha)$-variation function in $[a, b]$, then $f$ is an $\alpha$-Lipschitz function.

Proof. If $f \in \mathrm{BV}_{(2, \alpha)}[a, b]=\mathrm{BV}^{(2, \alpha)}[a, b]$ from Lemma 11 there exists $M>0$ such that for all $a \leqslant x<y \leqslant b$ we have that $\left|f_{\alpha}[x, y]\right| \leqslant M$ holds. Then by the definition of $f_{\alpha}$, we get $|f(y)-f(x)| \leqslant M|\alpha(y)-\alpha(x)|$; that is, $f$ is an $\alpha$-Lipschitz function in $[a, b]$.

Corollary 18. If $f \in \mathrm{BV}_{(2, \alpha)}[a, b]=\operatorname{BV}^{(2, \alpha)}[a, b]$, then one has that $f$ is $\alpha$-absolutely continuous in $[a, b]$.

Corollary 19. If $f \in \mathrm{BV}_{(2, \alpha)}[a, b]=\mathrm{BV}^{(2, \alpha)}[a, b]$, then $f \in$ $\mathrm{BV}[a, b]$.

Corollary 20. If $f \in \mathrm{BV}_{(2, \alpha)}[a, b]=\mathrm{BV}^{(2, \alpha)}[a, b]$, then $f$ is (uniformly) continuous in $[a, b]$ and thus bounded in $[a, b]$.

The above results allow us to obtain the following chain of embeddings:

$$
\begin{aligned}
\mathrm{BV}_{(2, \alpha)}[a, b] & =\mathrm{BV}^{(2, \alpha)}[a, b] \subset \alpha \text {-Lip }[a, b] \\
& \subset \alpha-\mathrm{AC}[a, b] \subset \mathrm{C}[a, b] \subset \mathrm{B}[a, b] .
\end{aligned}
$$

Theorem 21. The set $\mathrm{BV}_{(2, \alpha)}[a, b]$ equipped with the sum of functions and the scalar product is a linear space.

Proof. Let $f, g \in \mathrm{BV}_{(2, \alpha)}[a, b]$ and $\Pi$ be a type I partition of $[a, b]$. Then by the triangle inequality, we obtain $\sigma_{(2, \alpha)}(f+$ $g, \Pi) \leqslant \sigma_{(2, \alpha)}(f, \Pi)+\sigma_{(2, \alpha)}(g, \Pi)$ and thus $\mathrm{V}_{(2, \alpha)}(f+g) \leqslant$ 
$\mathrm{V}_{(2, \alpha)}(f)+\mathrm{V}_{(2, \alpha)}(g)$ and from this we conclude that $f+g \in$ $\mathrm{BV}_{(2, \alpha)}[a, b]$. Moreover $\sigma_{(2, \alpha)}(\lambda f, \Pi)=|\lambda| \sigma_{(2, \alpha)}(f), \lambda \in \mathbb{R}$, and $\mathrm{V}_{(2, \alpha)}(\lambda f)=|\lambda| \mathrm{V}_{(2, \alpha)}(f)$ from which we obtain that if $f \in \mathrm{BV}_{(2, \alpha)}[a, b]$, then $\lambda f \in \mathrm{BV}_{(2, \alpha)}[a, b], \lambda \in \mathbb{R}$.

Corollary 22. One has that

(1) $\left(\mathrm{BV}^{(2, \alpha)}[a, b],+, \cdot\right)$ is a real linear space;

(2) $\left(\mathrm{BV}_{(2, \alpha)}[a, b],+, \cdot\right)$ is a real linear space.

\section{Jordan Type Decomposition}

In this section we want to obtain a decomposition theorem in the spirit of Proposition 1. Before doing that, we want to give the following lemma, which is of direct verification.

Lemma 23. Let $f \in \mathrm{BV}_{(2, \alpha)}[a, b]$. If $a<c<b$

$$
\mathrm{V}_{(2, \alpha)}(f,[a, c])+\mathrm{V}_{(2, \alpha)}(f,[c, b]) \leqslant \mathrm{V}_{(2, \alpha)}(f,[a, b]) \text {. }
$$

We have the following decomposition theorem for functions in $\mathrm{BV}_{(2, \alpha)}$.

Theorem 24. One has that $f \in \mathrm{BV}_{(2, \alpha)}[a, b]$ if and only if $f=$ $f_{1}-f_{2}$, where $f_{1}$ and $f_{2}$ are $\alpha$-convex functions.

Proof. Observe that the set $E=\left\{x \in[a, b]: f_{\alpha}^{\prime}(x)\right.$ does not exist $\}$ has measure zero. For $x \in[a, b] \backslash E$ let us define

$$
\begin{aligned}
& p(x)=\frac{1}{2}\left\{\mathrm{~V}_{(2, \alpha)}(f,[a, x])+f_{\alpha}^{\prime}(x)\right\}, \\
& q(x)=\frac{1}{2}\left\{\mathrm{~V}_{(2, \alpha)}(f,[a, x])-f_{\alpha}^{\prime}(x)\right\}
\end{aligned}
$$

then $f_{\alpha}^{\prime}(x)=p(x)-q(x)$. Using Lemma 23 we observe that for $y<x$ we have

$$
\begin{aligned}
2[p(x)-p(y)]= & \mathrm{V}_{(2, \alpha)}(f,[a, x]) \\
& -\mathrm{V}_{(2, \alpha)}(f,[a, y])+f_{\alpha}^{\prime}(x) \\
& -f_{\alpha}^{\prime}(y) \\
\geq & \mathrm{V}_{(2, \alpha)}(f,[y, x]) \\
& -\left|f_{\alpha}^{\prime}(x)-f_{\alpha}^{\prime}(y)\right| \geq 0
\end{aligned}
$$

and a similar calculation can be made regarding the function $q$ and therefore $p$ and $q$ are increasing in $[a, b] \backslash E$. Since $f \in$ $\alpha$-AC $[a, b]$, then $f_{\alpha}^{\prime}$ is integrable in the sense of LebesgueStieltjes and $f(x)=f(a)+(\mathrm{LS}) \int_{a}^{x} f_{\alpha}^{\prime}(t) d \alpha(t)$, for $x \in[a, b]$. In $E$ we define $p$ and $q$ such that $p$ and $q$ are increasing and bounded in $[a, b]$ and therefore LS-integrable in $[a, b]$ with respect to $\alpha$; from Theorem 8 we obtain

$$
\begin{aligned}
f(x) & =(\mathrm{LS}) \int_{a}^{x} f_{\alpha}^{\prime}(t) d \alpha(t)+f(a) \\
& =(\mathrm{LS}) \int_{a}^{x} p(t) d \alpha(t)-(\mathrm{LS}) \int_{a}^{x} q(t) d \alpha(t) .
\end{aligned}
$$

Since each integral is $\alpha$-convex (see Theorem 9), the result follows.

Conversely, suppose that $f=f_{1}-f_{2}$ where $f_{1}$ and $f_{2}$ are $\alpha$-convex functions. We are going to prove that $f \in$ $\operatorname{BV}_{(2, \alpha)}[a, b]$. To do so, it is enough to verify that all $\alpha$-convex functions have second bounded $\alpha$-variation.

Let $v:[a, b] \rightarrow \mathbb{R}$ be $\alpha$-convex; then by Lemma 10 we have $v_{\alpha+}^{\prime}(a) \leqslant v_{a}[x, y] \leqslant v_{\alpha-}^{\prime}(b)$. Let $\Pi=\left\{a=x_{1}<x_{2}<\right.$ $\left.\cdots<x_{n}=b\right\}$ be a partition of type I of the interval $[a, b]$. Since $v_{\alpha}[\cdot, \cdot]$ is increasing we obtain

$$
\begin{aligned}
\sigma_{(2, \alpha)}(v, \Pi) & =\sum_{j=1}^{n-2}\left|v_{\alpha}\left[x_{j}, x_{j+1}\right]-v_{\alpha}\left[x_{j+1}, x_{j+2}\right]\right| \\
& =\sum_{j=1}^{n-2} v_{\alpha}\left[x_{j}, x_{j+1}\right]-v_{\alpha}\left[x_{j+1}, x_{j+2}\right] .
\end{aligned}
$$

This amount is part of a telescopic series; one more time by Lemma 10 we have

$$
\begin{aligned}
\sigma_{(2, \alpha)}(v, \Pi) & =v_{\alpha}\left[b, x_{n-1}\right]-v_{\alpha}\left[a, x_{2}\right] \\
& \leqslant v_{\alpha-}^{\prime}(b)-v_{\alpha+}^{\prime}(a)<\infty .
\end{aligned}
$$

Hence $\mathrm{V}_{(2, \alpha)(v)}<\infty$ and $v \in \mathrm{BV}_{(2, \alpha)}[a, b]$. Since $f_{1}$ and $f_{2}$ are $\alpha$-convex, therefore $f=f_{1}-f_{2} \in \mathrm{BV}_{(2, \alpha)}[a, b]$.

From Theorem 24 we deduce the following.

Corollary 25. If $f \in \mathrm{BV}_{(2, \alpha)}[a, b]$, then $f$ can be written as

$$
f(x)=(\mathrm{LS}) \int_{a}^{x} g(t) d \alpha(t)+f(a)
$$

where $g$ is a bounded variation function in $[a, b]$.

As another consequence of Theorem 24 and the $\alpha$-convex property, we derive the existence of $f_{\alpha+}^{\prime}\left(x_{0}\right)$ and $f_{\alpha-}^{\prime}\left(x_{0}\right)$ in each point $x_{0} \in(a, b)$ and $f_{\alpha+}^{\prime}(a), f_{\alpha_{-}}^{\prime}(b)$.

\section{6. $\mathrm{BV}^{(2, \alpha)}[a, b]=\mathrm{BV}_{(2, \alpha)}[a, b]$ as Banach Spaces}

Corollary 22 shows us that $\left(\mathbb{R}, \mathrm{BV}_{(2, \alpha)}[a, b],+, \cdot\right)$ is a linear space. Now we introduce a norm in this space.

Definition 26. Let $f \in \mathrm{BV}_{(2, \alpha)}[a, b]$. One defines $\|\cdot\|_{\mathrm{BV}_{(2, \alpha)}[a, b]}$ as

$$
\|f\|_{\mathrm{BV}_{(2, \alpha)}[a, b]}=|f(a)|+\left|f_{\alpha}^{\prime}(a)\right|+\mathrm{V}_{(2, \alpha)}(f) .
$$

When there is no danger of confusion, one will denote $\|\cdot\|_{\mathrm{BV}_{(2, \alpha)}[a, b]}=:\|\cdot\|_{(2, \alpha)}$.

Lemma 27. If $f \in \mathrm{BV}_{(2, \alpha)}[a, b]$ and $\mathrm{V}_{(2, \alpha)}(f)=0$, then $f(x)$ $=\lambda \alpha(x)+\mu$, where $\lambda, \mu \in \mathbb{R}$.

Proof. Note that $\mathrm{V}_{(2, \alpha)}(f)=0$ if and only if $\sigma_{(2, \alpha)}(f)=0$ for all partitions $\Pi$ of $[a, b]$. Consider the particular partition 
$\Pi_{0}=\{a<x<b\}$. Then from the fact that $\sigma_{(2, \alpha)}\left(f, \Pi_{0}\right)=0$ we deduce

$$
f(x)=\frac{f(b)-f(a)}{\alpha(b)-\alpha(a)} \alpha(x)+\frac{f(a) \alpha(b)-f(b) \alpha(a)}{\alpha(b)-\alpha(a)} .
$$

Taking

$$
\begin{aligned}
& \lambda=\frac{f(b)-f(a)}{\alpha(b)-\alpha(a)} \\
& \mu=\frac{f(a) \alpha(b)-f(b) \alpha(a)}{\alpha(b)-\alpha(a)}
\end{aligned}
$$

the result follows.

Theorem 28. The functional $\|\cdot\|_{\mathrm{BV}_{(2, \alpha)}[a, b]}=\|\cdot\|_{(2, \alpha)}$ is a norm in the space $\mathrm{BV}_{(2, \alpha)}[a, b]$.

Proof. Let $f, g \in \mathrm{BV}_{(2, \alpha)}[a, b]$. We have

$$
\begin{aligned}
\|f+g\|_{(2, \alpha)}= & |(f+g)(a)|+\left|(f+g)_{\alpha}^{\prime}(a)\right| \\
& +\mathrm{V}_{(2, \alpha)}(f+g) \\
\leqslant & |f(a)|+|g(a)|+\left|f_{\alpha}^{\prime}(a)\right|+\left|g_{\alpha}^{\prime}(a)\right| \\
& +\mathrm{V}_{(2, \alpha)}(f)+\mathrm{V}_{(2, \alpha)}(g) \\
= & \|f\|_{(2, \alpha)}+\|g\|_{(2, \alpha)} .
\end{aligned}
$$

Let $\lambda \in \mathbb{R}, f \in \mathrm{BV}_{(2, \alpha)}[a, b]$; we get $\|\lambda f\|_{(2, \alpha)}=|\lambda|\|f\|_{(2, \alpha)}$. Finally $\|f\|_{(2, \alpha)}=0$ if and only if $|f(a)|=0$ and $\left|f_{\alpha}^{\prime}(a)\right|=0$ and $\mathrm{V}_{(2, \alpha)}(f)=0$. Since $\mathrm{V}_{(2, \alpha)}(f)=0$, from Lemma 27 we have $f(x)=\lambda \alpha(x)+\mu, \lambda, \mu \in \mathbb{R}$. Moreover $f_{\alpha}^{\prime}(x)=\lambda$, but $f_{\alpha}^{\prime}(a)=0$; then $\lambda=0$. Thus $f(x)=\mu$, but $f_{\alpha}^{\prime}(a)=0$; then $\mu=0$. Hence $f=0$.

In this way we have shown that $\left(\mathbb{R}, \operatorname{BV}_{(2, \alpha)}[a, b],+, \cdot\right.$, $\left.\|\cdot\|_{(2, \alpha)}\right)$ is a normed space.

Now, we are going to prove that this space is complete. In Theorem 17 we showed that $\mathrm{BV}_{(2, \alpha)}[a, b] \subset \alpha$-Lip $[a, b]$. In what follows we prove that this inclusion is a normed space embedding to conclude that $\mathrm{BV}_{(2, \alpha)}[a, b]$ is complete.

Lemma 29. Let $f \in \mathrm{BV}_{(2, \alpha)}[a, b]$; then

$$
\|f\|_{\alpha-\operatorname{Lip}[a, b]} \leqslant\|f\|_{\mathrm{BV}_{(2, \alpha)}[a, b]} .
$$

Proof. Let us consider $a<a+h<s<t<b$; then from Theorem 14 we obtain

$$
\begin{gathered}
\left|\frac{f(t)-f(s)}{\alpha(t)-\alpha(s)}-\frac{f(a+h)-f(a)}{\alpha(a+h)-\alpha(a)}\right| \\
\leqslant \mathrm{V}^{(2, \alpha)}(f) \leqslant \mathrm{V}_{(2, \alpha)}(f) .
\end{gathered}
$$

Letting $h \rightarrow 0$ we have

$$
\left|\frac{f(t)-f(s)}{\alpha(t)-\alpha(s)}-f_{\alpha}^{\prime}(a)\right| \leqslant \mathrm{V}_{(2, \alpha)}(f) ;
$$

thus

$$
\begin{aligned}
& \left|\frac{f(t)-f(s)}{\alpha(t)-\alpha(s)}\right| \leqslant\left|f_{\alpha}^{\prime}(a)\right|+\mathrm{V}_{(2, \alpha)}(f), \\
& |f(a)|+\left|\frac{f(t)-f(s)}{\alpha(t)-\alpha(s)}\right| \\
& \leqslant|f(a)|+\left|f_{\alpha}^{\prime}(a)\right|+\mathrm{V}_{(2, \alpha)}(f) ;
\end{aligned}
$$

from this we get

$$
|f(a)|+\sup _{s \neq t}\left|\frac{f(t)-f(s)}{\alpha(t)-\alpha(s)}\right| \leqslant\|f\|_{(2, \alpha)} .
$$

That is, $\|f\|_{\alpha-\operatorname{Lip}[a, b]} \leqslant\|f\|_{(2, \alpha)}$.

Theorem 30. ( $\left.\mathbb{R}, \mathrm{BV}_{(2, \alpha)}[a, b],+, \cdot,\|\cdot\|_{(2, \alpha)}\right)$ is a Banach space.

Proof. Let $\left\{f_{p}\right\}_{p \in \mathbb{N}}$ be a Cauchy sequence in $\mathrm{BV}_{(2, \alpha)}[a, b]$. Given $\varepsilon>0$ there exists $N_{\varepsilon} \in \mathbb{N}$ such that if $p, q>N_{\varepsilon}$ then $\left\|f_{p}-f_{q}\right\|_{(2, \alpha)}<\varepsilon$. By the definition of the norm, we obtain $\left|f_{p}(a)-f_{q}(a)\right|<\varepsilon,\left|\left(f_{p}\right)_{\alpha}^{\prime}-\left(f_{q}\right)_{\alpha}^{\prime}\right|<\varepsilon$ and $\mathrm{V}_{(2, \alpha)}\left(f_{p}-f_{q}\right)<\varepsilon$. Invoking Lemma 29 it follows that $\left\|f_{p}-f_{q}\right\|_{\alpha \text {-Lip }[a, b]} \leqslant \| f_{p}-$ $f_{q} \|_{(2, \alpha)}<\varepsilon$ and thus $\left\{f_{p}\right\}_{p \in \mathbb{N}}$ is a Cauchy sequence in $\alpha$ Lip $[a, b]$. In other words

$$
\begin{aligned}
& \left|f_{p}(a)-f_{q}(a)\right| \\
& \quad+\sup _{x \neq y}\left|\frac{\left(f_{p}-f_{q}\right)(x)-\left(f_{p}-f_{q}\right)(y)}{\alpha(x)-\alpha(y)}\right|<\varepsilon,
\end{aligned}
$$

which implies that $\left|f_{p}(a)-f_{q}(a)\right|<\varepsilon$ and $\mid\left(\left(f_{p}-f_{q}\right)(x)-\right.$ $\left.\left(f_{p}-f_{q}\right)(y)\right) /(\alpha(x)-\alpha(y)) \mid<\varepsilon$ for $x \in[a, b]$. By the triangle inequality we have $\left|f_{p}(x)-f_{q}(x)\right|<\varepsilon(1+\alpha(b)-\alpha(a))$, for $x \in[a, b]$. This tells us that $\left\{f_{p}(x)\right\}_{p \in \mathbb{N}}$ is a Cauchy sequence in $\mathbb{R}$. Since $\mathbb{R}$ is complete we can define a function $f$ in the following way: $f:[a, b] \rightarrow \mathbb{R}$ given by $f(x)=$ $\lim _{p \rightarrow \infty} f_{p}(x)$, for $x \in[a, b]$. Next we need to prove that (I) $f \in \mathrm{BV}_{(2, \alpha)}[a, b]$ and (II) $\left\{f_{p}\right\}_{p \in \mathbb{N}}$ converges to $f$ in $\|\cdot\|_{(2, \alpha)^{-}}$ norm.

(I) Indeed, let $\Pi=\left\{a=x_{1}<x_{2}<\cdots<x_{n}=b\right\}$ be a partition of $[a, b]$. Then

$$
\begin{aligned}
& \sum_{j=1}^{n-2}\left|\frac{f_{p}\left(x_{j+2}\right)-f_{p}\left(x_{j+1}\right)}{\alpha\left(x_{j+2}\right)-\alpha\left(x_{j+1}\right)}-\frac{f_{p}\left(x_{j+1}\right)-f_{p}\left(x_{j}\right)}{\alpha\left(x_{j+1}\right)-\alpha\left(x_{j}\right)}\right| \\
& \quad \leqslant\left\|f_{p}\right\|_{(2, \alpha)}<M
\end{aligned}
$$

for all $p \in \mathbb{N}$ and partition $\Pi$ (the sequence $\left\{\left\|f_{p}\right\|_{(2, \alpha)}\right\}_{p \in \mathbb{N}}$ is bounded since $\left\{f_{p}\right\}_{p \in \mathbb{N}}$ is a Cauchy sequence). 
Now,

$$
\begin{aligned}
& \lim _{p \rightarrow \infty} \sum_{j=1}^{n-2} \mid \frac{f_{p}\left(x_{j+2}\right)-f_{p}\left(x_{j+1}\right)}{\alpha\left(x_{j+2}\right)-\alpha\left(x_{j+2}\right)} \\
& -\frac{f_{p}\left(x_{j+1}\right)-f_{p}\left(x_{j}\right)}{\alpha\left(x_{j+1}\right)-\alpha\left(x_{j}\right)}\left|=\sum_{j=1}^{n-2}\right| \frac{f\left(x_{j+2}\right)-f\left(x_{j+1}\right)}{\alpha\left(x_{j+2}\right)-\alpha\left(x_{j+2}\right)} \\
& -\frac{f\left(x_{j+1}\right)-f\left(x_{j}\right)}{\alpha\left(x_{j+1}\right)-\alpha\left(x_{j}\right)} \mid \leqslant M
\end{aligned}
$$

for all partitions $\Pi$; therefore $\mathrm{V}_{(2, \alpha)}(f) \leqslant M$; thus $f \in$ $\mathrm{BV}_{(2, \alpha)}[a, b]$. Also note that $f_{\alpha}^{\prime}(a)$ exists.

(II) Let $\Pi=\left\{a=x_{1}<x_{2}<\cdots<x_{n}=b\right\}$ be a partition of $[a, b]$. For $p, q>N_{\varepsilon}$ we obtain

$$
\begin{aligned}
& \sum_{j=1}^{n-2} \mid \frac{\left(f_{p}-f_{q}\right)\left(x_{j+2}\right)-\left(f_{p}-f_{q}\right)\left(x_{j+1}\right)}{\alpha\left(x_{j+2}\right)-\alpha\left(x_{j+1}\right)} \\
& -\frac{\left(f_{p}-f_{q}\right)\left(x_{j+1}\right)-\left(f_{p}-f_{q}\right)\left(x_{j}\right)}{\alpha\left(x_{j+1}\right)-\alpha\left(x_{j}\right)} \mid \leqslant \mathrm{V}_{(2, \alpha)}\left(f_{p}\right. \\
& \left.-f_{q}\right)<\varepsilon .
\end{aligned}
$$

Taking limit as $q$ goes to infinity, we have

$$
\begin{gathered}
\sum_{j=1}^{n-2} \mid \frac{\left(f_{p}-f\right)\left(x_{j+2}\right)-\left(f_{p}-f\right)\left(x_{j+1}\right)}{\alpha\left(x_{j+2}\right)-\alpha\left(x_{j+2}\right)} \\
-\frac{\left(f_{p}-f\right)\left(x_{j+1}\right)-\left(f_{p}-f\right)\left(x_{j}\right)}{\alpha\left(x_{j+1}\right)-\alpha\left(x_{j}\right)} \mid \leqslant \varepsilon,
\end{gathered}
$$

which holds for any partition of $[a, b]$; then $\mathrm{V}_{(2, \alpha)}\left(f_{p}-f\right) \leqslant \varepsilon$ if $p>N_{\varepsilon}$. Since $\left|f_{p}(a)-f_{q}(a)\right|<\varepsilon$ we have $\left|f_{p}(a)-f(a)\right| \leqslant \varepsilon$, for $p>N_{\varepsilon}$, if $q \rightarrow \infty$.

Next, for $a<a+h<s<t<b$, we have

$$
\begin{aligned}
& \mid \frac{\left(f_{p}-f\right)(t)-\left(f_{p}-f\right)(s)}{\alpha(t)-\alpha(s)} \\
& \quad-\frac{\left(f_{p}-f\right)(a+h)-\left(f_{p}-f\right)(a)}{\alpha(a+h)-\alpha(a)} \mid \leqslant \mathrm{V}^{(2, \alpha)}\left(f_{p}\right. \\
& -f) \leqslant \mathrm{V}_{(2, \alpha)}\left(f_{p}-f\right) \leqslant \varepsilon
\end{aligned}
$$

if $p>N_{\varepsilon}$. Taking limit as $h$ goes to zero

$$
\begin{aligned}
& \left|\frac{\left(f_{p}-f\right)(t)-\left(f_{p}-f\right)(s)}{\alpha(t)-\alpha(s)}-\left(f_{p}\right)_{\alpha}^{\prime}(a)+f_{\alpha}^{\prime}(a)\right| \\
& \leqslant \varepsilon
\end{aligned}
$$

if $p>N_{\varepsilon}$. Since $f_{p}, f \in \mathrm{BV}_{(2, \alpha)}[a, b] \subset \alpha$-Lip $[a, b]$

$$
\begin{aligned}
& \left|\left(f_{p}\right)_{\alpha}^{\prime}(a)-f_{\alpha}^{\prime}(a)\right| \\
& \quad \leqslant \varepsilon+\left|\frac{\left(f_{p}-f\right)(t)-\left(f_{p}-f\right)(s)}{\alpha(t)-\alpha(s)}\right| \\
& \quad \leqslant \varepsilon+\left\|f_{p}-f\right\|_{\alpha-\operatorname{Lip}[a, b]} \leqslant 2 \varepsilon
\end{aligned}
$$

if $p>N_{\varepsilon}$, by virtue of $\left\|f_{p}-f\right\|_{\alpha-\operatorname{Lip}[a, b]}=\lim _{q \rightarrow \infty} \| f_{p}-$ $f_{q} \|_{\alpha-\operatorname{Lip}[a, b]}<\varepsilon$. Finally, for $p>N_{\varepsilon}$ we have $\left|f_{p}(a)-f(a)\right|+$ $\left|\left(f_{p}\right)_{\alpha}^{\prime}(a)-f_{\alpha}^{\prime}(a)\right|+\mathrm{V}_{(2, \alpha)}\left(f_{p}-f\right)<4 \varepsilon$, that is, $\left\|f_{p}-f\right\|_{(2, \alpha)}<$ $4 \varepsilon$.

\section{7. $B V_{(2, \alpha)}$ as a Banach Algebra}

As a result of the generalized Orlicz-Maligranda inequality proved in [15], we can show that the $\mathrm{BV}_{(2, \alpha)}$ is a Banach algebra, as was done in [16]; namely, consider the following.

Theorem 31. The space $\mathrm{BV}_{(2, \alpha)}([a, b])$ equipped with the norm

$$
\begin{aligned}
\|f\|_{\mathrm{BV}_{(2, \alpha)}([a, b])}^{1}= & \|f\|_{\infty} \\
& +2(\alpha(b)-\alpha(a))\|f\|_{\mathrm{BV}_{(2, \alpha)}([a, b])}, \\
& \quad f \in \mathrm{BV}_{(2, \alpha)}([a, b])
\end{aligned}
$$

is a Banach algebra and the norms $\|\cdot\|_{\mathrm{BV}_{(2, \alpha)}[a, b]}$ and $\|\cdot\|_{\mathrm{BV}}^{1}$ are equivalent.

\section{Conflict of Interests}

The authors declare that there is no conflict of interests regarding the publication of this paper.

\section{Acknowledgments}

The authors would like to thank the anonymous referee for careful reading of the paper. The second named author was partially supported by Pontificia Universidad Javeriana under the research project Study of Boundedness Variation in the Sense of de La Vallée Poussin (ID PRY: 006780, ID PPTA: 006654).

\section{References}

[1] C. Jordan, "Sur la série de Fourier," Comptes Rendus de l'Académie des Sciences, Paris, vol. 2, pp. 228-230, 1881.

[2] S. I. Hudjaev and A. I. Vol'pert, Analysis in Classes of Discontinuous Functions and Equations of Mathematical Physics, Springer, 1985.

[3] J. Appell, J. Banas, and N. Merentes, Bounded Variation and Around, De Gruyter, 2013.

[4] R. E. Castillo, H. Rafeiro, and E. Trousselot, "A generalization for the Riesz p-variation," Revista Colombiana de Matemáticas, vol. 48, no. 2, pp. 165-190, 2014. 
[5] R. E. Castillo, H. Rafeiro, and E. Trousselot, "Embeddings on spaces of generalized bounded variation," Revista Colombiana de Matemáticas, vol. 48, no. 1, pp. 97-109, 2014.

[6] R. E. Castillo and E. Trousselot, "On functions of ( $p, \alpha)$-bounded variation," Real Analysis Exchange, vol. 34, no. 1, pp. 49-60, 2008.

[7] J. C. de La Vallée Poussin, "Sur la convergence des formules d'interpolation entre ordonnées équidistantes," Bulletins de l'Académie Royale des Sciences, pp. 319-410, 1908.

[8] H. L. Royden, Real Analysis, Collier Macmillan, London, UK, 3rd edition, 1988.

[9] A. M. Russell, "Functions of bounded second variation and Stieltjes-type integrals," Journal of the London Mathematical Society. Second Series, vol. 2, pp. 193-208, 1970.

[10] T. Popoviciu, "Sur quelques propriétés des fonctions d'une variable réelle convexes d'ordre supérieur," Buletinul Societatii de Stiinte din Cluj, vol. 7, pp. 254-282, 1933.

[11] M. C. Chakvabarty, "Some result on AC-w-functions," Fundamenta Mathematica, vol. 64, pp. 219-230, 1969.

[12] P. Halmos, Measure Theory, Springer, 1974.

[13] R. L. Jeffery, "Generalized integrals with respect to functions of bounded variation," Canadian Journal of Mathematics, vol. 10, pp. 617-626, 1958.

[14] F. Riesz and B. Nagy, Functional Analysis, Ungar, New York, NY, USA, 1955, Translated from the Second French Edition.

[15] R. E. Castillo and E. Trousselot, "A generalization of the Maligranda-Orlicz lemma," Journal of Inequalities in Pure and Applied Mathematics, vol. 8, no. 4, article 115, p. 3, 2007.

[16] R. E. Castillo and E. Trousselot, "An application of the generalized Maligranda-Orlicz's Lemma," Journal of Inequalities in Pure and Applied Mathematics, vol. 9, no. 3, article 84, 2008. 


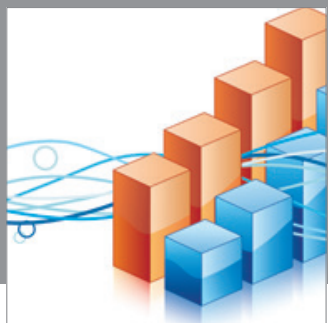

Advances in

Operations Research

mansans



The Scientific World Journal
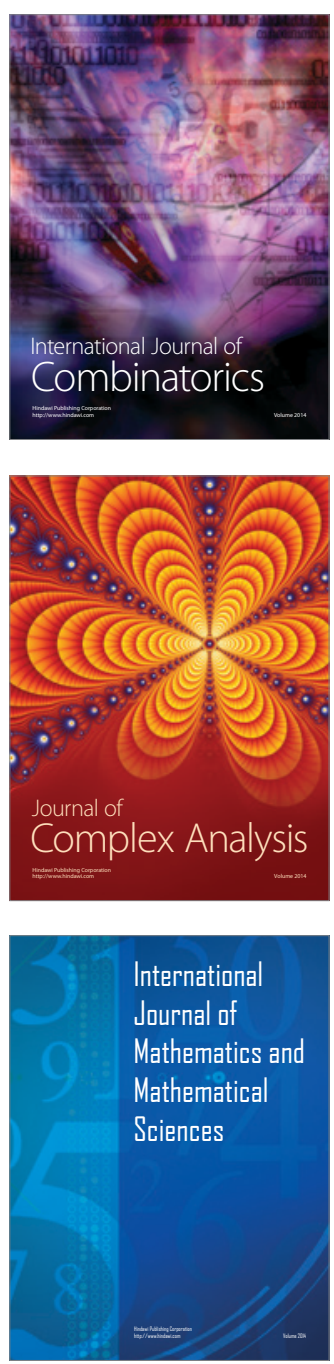
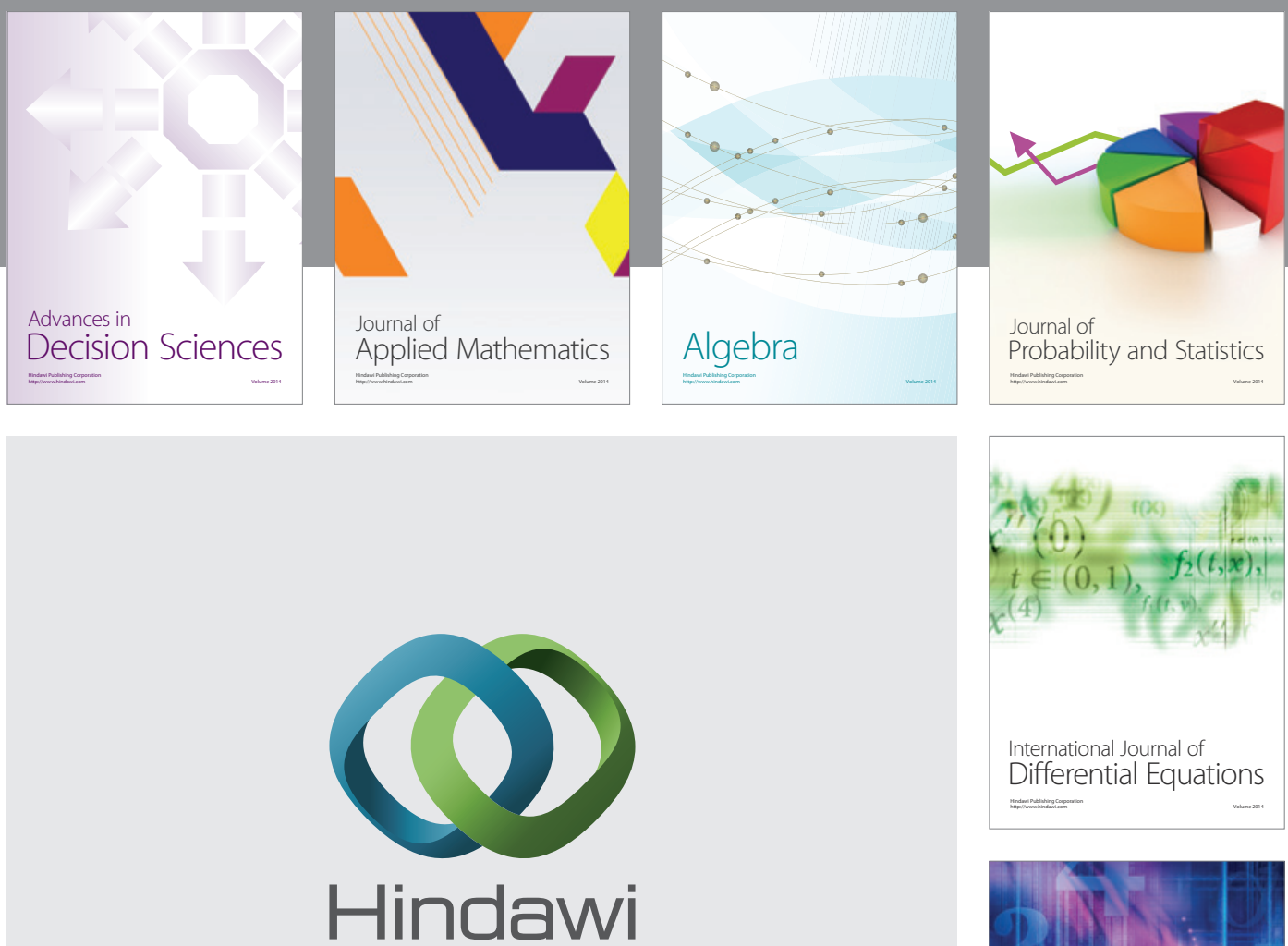

Submit your manuscripts at http://www.hindawi.com
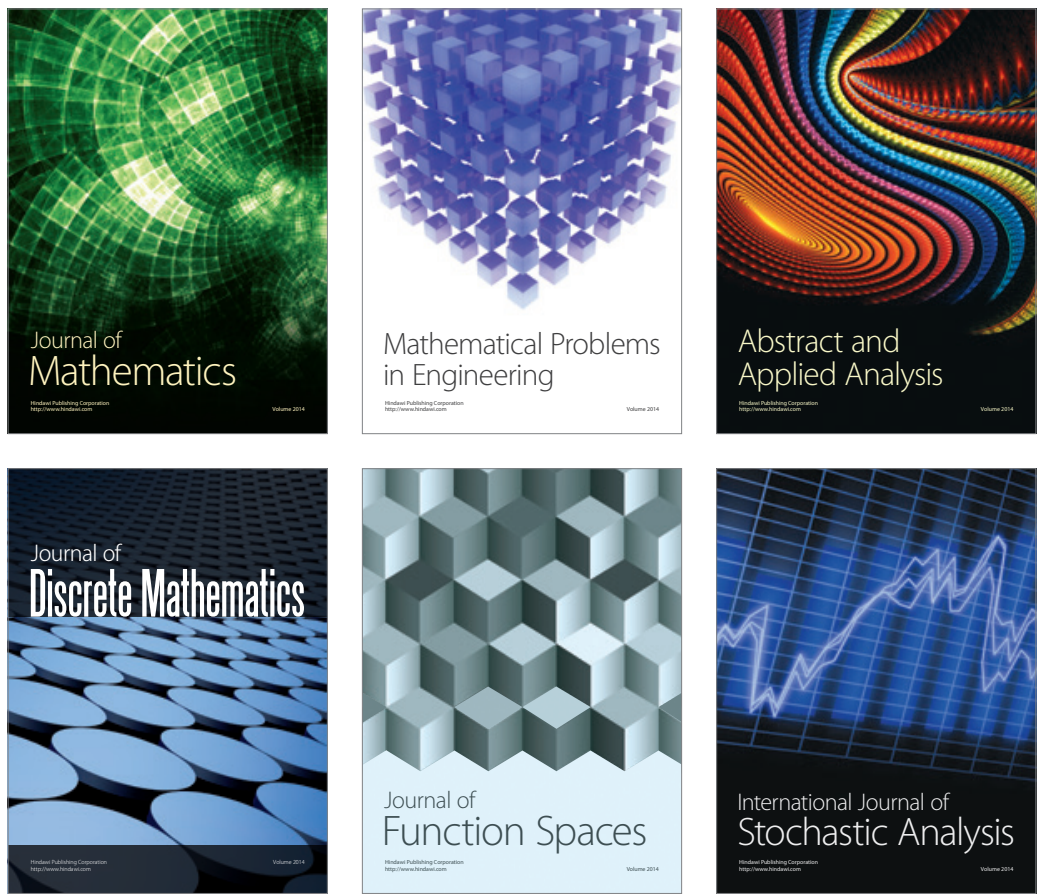

Journal of

Function Spaces

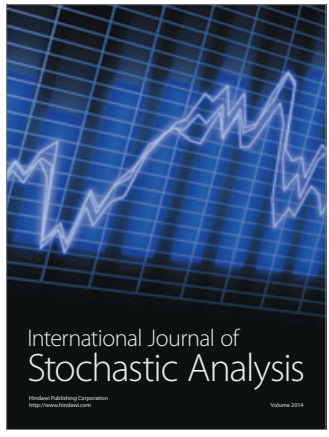

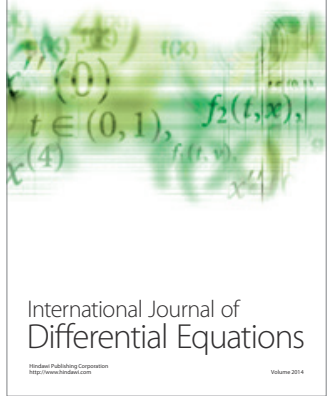
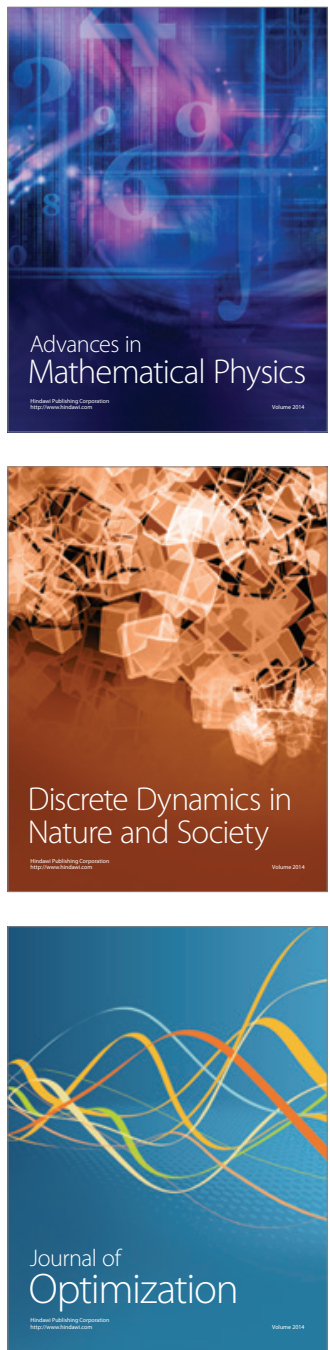